\title{
Understanding the evolution dynamics of internet topology
}

\author{
Shi Zhou* \\ University College London, Adastral Park Campus, Ross Building, Ipswich, IP5 3RE, United Kingdom
}

(Received 2 December 2005; revised manuscript received 18 April 2006; published 31 July 2006)

\begin{abstract}
The internet structure is extremely complex. The positive-feedback preference (PFP) model is a recently introduced internet topology generator. The model uses two generic algorithms to replicate the evolution dynamics observed on the internet historic data. The phenomenological model was originally designed to match only two topology properties of the internet, i.e., the rich-club connectivity and the exact form of degree distribution, whereas numerical evaluation has shown that the PFP model accurately reproduces a large set of other nontrivial characteristics as well. This paper aims to investigate why and how this generative model captures so many diverse properties of the internet. Based on comprehensive simulation results, the paper presents a detailed analysis on the exact origin of each of the topology properties produced by the model. This work reveals how network evolution mechanisms control the obtained topology properties and it also provides insights on correlations between various structural characteristics of complex networks.
\end{abstract}

DOI: 10.1103/PhysRevE.74.016124

PACS number(s): 89.75.-k, 87.23.Ge, 05.70.Ln

\section{INTRODUCTION}

It is vital to obtain a good description of a network topology because structure fundamentally affects function $[1,2]$. The internet contains millions of routers, which are grouped into thousands of subnetworks, called autonomous systems (ASs), which are then glued into a global network by the Border Gateway Protocol. Effective engineering of the internet is predicated on a detailed understanding of issues such as the large-scale structure of its underlying physical topology, the manner in which it evolves over time, and the way in which its constituent components contribute to its overall function [3].

The recently introduced positive-feedback preference (PFP) model [4] is an internet AS-level topology generator. The model uses two evolution mechanisms, namely the interactive growth and the positive-feedback preference. Both mechanisms are inspired by, and coincident with, practical observations on the internet historic data. Originally the phenomenological model was designed to match only the internet's rich-club connectivity [5] and the exact form of degree distribution, including the distribution of low degrees, the maximum degree, and the degree distribution's power-law exponent [6]. It has been a pleasant surprise that numerical evaluation against the internet measurement date has shown that the model accurately reproduces a large set of other nontrivial topology characteristics as well, including the disassortative mixing [7-9], the shortest path length [10], short cycles $[11,12]$, and the betweenness centrality [13]. The PFP model has been used in more realistic simulations of the internet, e.g. Ref. [14].

As of this writing, an analytical solution of the PFP model is not available yet. In this paper we use the numerical method to analyze why and how the model is able to reproduce a fuller picture of the internet than it was designed for. In other words, we aim to investigate the exact origin of each of the topology properties captured by the PFP model. An-

\footnotetext{
*E-mail address: s.zhou@adastral.ucl.ac.uk
}

swers to these questions can be valuable for the ongoing effort on a mathematical solution of the model.

In Sec. II, we review the PFP model and its two mechanisms. We reflect on the intuitions underlying the design of the two evolution mechanisms. In Sec. III, we comparatively examine two limiting cases of the PFP model. Based on detailed numerical simulations, we identify the explicit evolution dynamics that are responsible for generating the richclub phenomenon and the degree distribution properties. We also reveal that the origin of the disassortative mixing is in fact already embedded in the model's two evolution mechanisms. Furthermore, we explain that the PFP model resembles the internet's shortest path length and short cycles because these two characteristics are correlated with other topology properties.

In Sec. IV, the above results become more evident when we examine how the PFP model's topology properties react to the change of the parameters that control the model's evolution mechanisms. Our investigation leads to a number of insightful discoveries. For example, we find out that the richclub connectivity is almost exclusively determined by the interactive growth mechanism. We also show that the interactive growth and the positive-feedback preference jointly contribute to the model's disassortative mixing behavior, however, the two mechanisms have opposite effects on the degree distribution's power-law exponent.

In Sec. V we conclude that this work represents a fuller and deeper understanding of the internet topology evolution dynamics. This work complements the research on evolution mechanisms and structural constraints of complex networks in general.

\section{POSITIVE-FEEDBACK PREFERENCE MODEL}

In graph theory, degree $k$ is defined as the number of links a node has. Degree is a local property but the distribution of degree provides a global view of a network structure. The degree distribution of the internet AS-level topology is characterized by a power law [6] as 

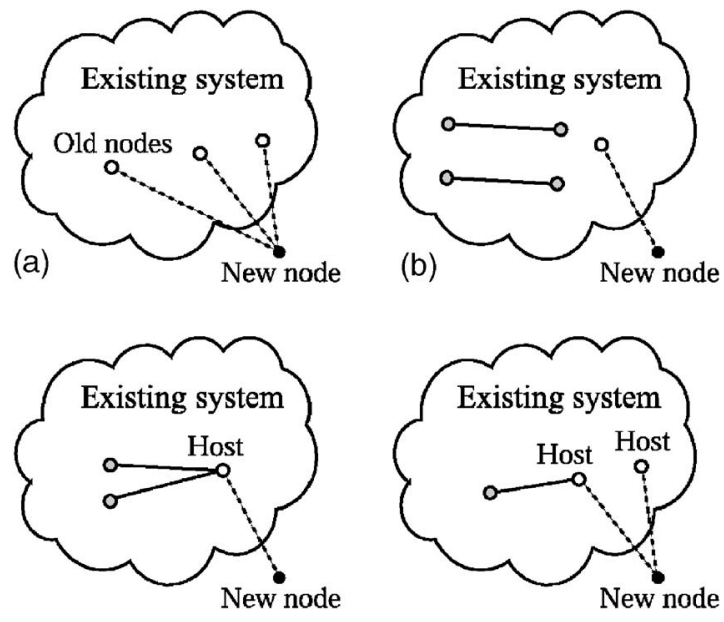

(c) with probability $p$

FIG. 1. Network growth mechanisms: (a) the BA model's newnode-only growth; (b) the independent growth; and (c) and (d) the PFP model's interactive growth.

$$
P(k) \sim k^{\gamma}, \quad \gamma \simeq-2.2 .
$$

A power-law degree distribution means the majority of nodes that form a network have very few links. Some nodes, however, are very well connected.

Barabási and Albert (BA) [15] showed that a power-law degree distribution can arise from two generic mechanisms: growth, where a network "grows" from a small random network by attaching new nodes to $m$ old nodes in the existing system [see Fig. 1(a), in which $m=3$ ]; and preferential attachment, where a new node is attached preferentially to nodes that are already well connected. The probability that a new node will be connected to a node with degree $k$ is given as

$$
\Pi(k)=\frac{k}{\sum_{j} k_{j}} .
$$

During the last few years a large number of models have been proposed to resemble and explain the power-law degree distribution and other topology properties observed on various real networks [1,2]. Recently Zhou and Mondragón [4] introduced the positive-feedback preference (PFP) model. Numerical evaluation has shown that the PFP model accurately reproduces a large number of characteristics of the internet AS-level topology. The model uses the following two evolution mechanisms.

\section{A. Mechanism one: Interactive growth}

The interactive growth is designed to satisfy a number of observations on internet history data [16-18]. First, the internet evolution is largely due to two processes: the attachment of new nodes to the existing system, and the addition of new internal links between old nodes already present in the existing system. Second, the majority of new nodes appearing on the internet are each attached to no more than two old nodes. And third, the ratio of links to nodes on the internet AS graph is approximately 3 .

Different from the independent growth [19] [see Fig. 1(b)], in which new nodes and new internal links are added independently, the two evolution processes are interdependent in the interactive growth [see Figs. 1(c) and 1(d)]. That is to say, a new internal link always starts from an old node, we call it a host node, to which a new node is attached. A heuristic explanation of this interaction is that on the internet, new customers (new nodes) generate extra demand for service, which triggers their service providers (host nodes) to develop new connections to other service providers (new internal links) in order to accommodate the increased traffic and improve services.

The interactive growth is described as follows: starting from a small random graph, at each time step,

(i) with probability $p \in[0,1]$, a new node is attached to a host node, and at the same time two new internal links are added connecting the host node to two other old nodes [see Fig. 1(c)];

(ii) with probability $1-p$, a new node is attached to two host nodes, and only one new internal link is added connecting one of the host nodes to another old node [see Fig. 1(d)].

Numerical simulation shows that when the probability parameter $p=0.4$, the interactive growth produces the best result.

\section{B. Mechanism two: positive-feedback preference}

The PFP model uses the following nonlinear preference probability to choose old nodes for the interactive growth:

$$
\Pi(k)=\frac{k^{1+\delta \ln k}}{\sum_{j} k_{j}^{1+\delta \ln k_{j}}}, \quad \delta \geq 0 .
$$

It is called the positive-feedback preference (PFP) because a node's ability to acquire new links increases as a feedback loop of the node's degree. When the parameter $\delta=0$, it is equivalent to the BA model's linear preference [see Eq. (2)]. Numerical simulation shows that the positive-feedback preference produces the best result when the parameter $\delta$ $=0.021[26]$.

\section{Discussion}

The positive-feedback preference is designed to satisfy the observation [16-18] that during the internet evolution, the probability that a new node links with a low-degree node follows the BA model's linear preference, whereas highdegree nodes have a stronger ability to acquire new links than predicted by the linear preference.

A preference probability can be written as $\Pi(k)$ $=f(k) / \Sigma_{j} f\left(k_{j}\right)$, where $f(k)$ is a degree function. The exponential preference $[20,21]$, which uses the nonlinear degree function of $f(k)=k^{\lambda}, \lambda \geq 1$, also gives high-degree nodes a stronger preference than the linear preference. However, as shown in Fig. 2, only the PFP can be approximated by the linear preference for low-degree nodes. When degree increases, the PFP grows more and more rapidly and finally exceeds the exponential preference. 


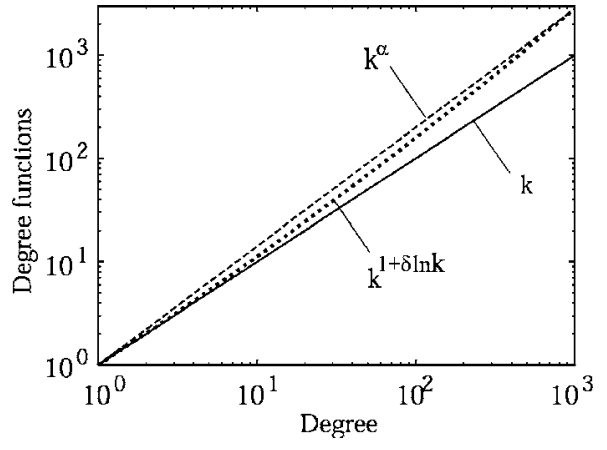

FIG. 2. Three degree functions: $f(k)=k$ for the linear preference, $f(k)=k^{1.15}$ for the exponential preference, and $f(k)=k^{1+0.021 \ln k}$ for the positive-feedback preference.

To illustrate the impact of such difference, we compare a $k$-degree node against a $\mu k$-degree node, $\mu \geqslant 1$. The ratio of their preference probability can be given as $\Pi(\mu k) / \Pi(k)$ $=f(\mu k) / f(k)$. As shown in Table I, for the linear and the exponential preferences, a 1000-degree node, when competing against a 100-degree node, has the same advantage as a 10 -degree node competing against a 1-degree node. Whereas for the PFP, $f(1000) / f(100)$ is more than $50 \%$ larger than $f(10) / f(1)$. This means the PFP not only appreciates the degree gap between low-degree nodes and high-degree nodes, but also effectively enlarges the degree difference between high-degree nodes themselves (see Fig. 3). The consequence of the positive-feedback preference is "the rich not only get richer, they get disproportionately richer."

\section{EVOLUTION MECHANISMS VS TOPOLOGY PROPERTIES}

To investigate the relations between the PFP model's evolution mechanisms and the obtained topology properties, we compare the PFP model and the BA model against the following two limiting cases:

(i) The interactive growth (IG) model, which uses the PFP model's interactive growth and the BA model's linear preference.

(ii) The BA+PFP model, which uses the BA model's new-node-only growth and the PFP model's positivefeedback preference.

For each of the four models, we generate ten networks to the same size as the internet AS graph [22,23] using different random seeds. All networks are generated from small ran-

TABLE I. Preference probability ratio $f(\mu k) / f(k)$ when $\lambda$ $=1.15$ and $\delta=0.021$.

\begin{tabular}{lccc}
\hline \hline & Linear & Exponential & Positive feedback \\
\hline$f(k)$ & $k$ & $k^{\lambda}$ & $k^{1+\delta \ln k}$ \\
$\frac{f(\mu k)}{f(k)}$ & $\mu$ & $\mu^{\lambda}$ & $\mu^{1+\delta \ln (\mu k)} k^{\delta \ln \mu}$ \\
$\frac{f(1000)}{f(100)}$ & $\frac{1000}{100}=10$ & $\frac{2818.4}{199.5}=14.1$ & $\frac{2722.7}{156.1}=17.4$ \\
$\frac{f(10)}{f(1)}$ & $\frac{10}{1}=10$ & $\frac{14.1}{1}=14.1$ & $\frac{11.2}{1}=11.2$ \\
\hline \hline
\end{tabular}

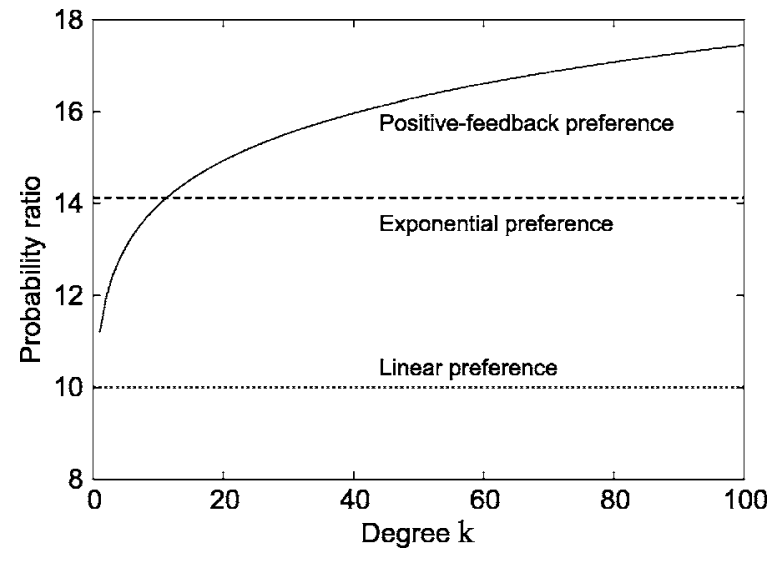

FIG. 3. Preference probability ratio $f(\mu k) / f(k)$ as a function of degree $k$ when $\mu=10, \lambda=1.15$, and $\delta=0.021$.

dom graphs consisting of ten nodes randomly connected by 30 links. Quantities in Table II are averages over the ten networks. Detailed evaluation of the PFP model against internet measurement data has been provided in Ref. [4]. In the following we focus on the comparison of topology properties among the four models.

\section{A. Rich-club phenomenon}

A hierarchical structure of the internet AS-level topology is the rich-club phenomenon [5], which describes the fact that well connected nodes, rich nodes, tend to be tightly interconnected with other rich nodes forming a core group, or club. Rich-club membership can be defined as "the $r$ best connected nodes," where $r$ is a node's rank denoting the node's position on the nonincreasing degree list of a network. Node rank is often normalized by $N$, the number of nodes contained in the network.

The rich-club phenomenon is assessed by measuring the rich-club connectivity $\varphi(r / N)$, defined as the ratio of the actual number of links to the maximum possible number of links among the rich-club members. The internet AS graph is fundamentally characterized by a power law of $\phi(r / N) \sim r^{\theta}$ with the exponent $\theta=-1.48$, which results from fitting $\phi(r / N)$ with a power law for $90 \%$ of the nodes, i.e., 0.1 $\leqslant r / N \leqslant 1$. Rich-club connectivity indicates how well club members "know" each other, e.g., $\varphi=1$ means that all the members have a direct link to any other member, i.e., they form a fully connected mesh, a clique. The top clique size $n_{\text {clique }}$ is defined as the maximum number of nodes with the highest ranks still forming a clique.

As shown in Fig. 4 and Table II, the PFP model and the IG model exhibit the same rich-club connectivity. So do the BA model and the BA+PFP model. However, the former two models, using the interactive growth, produce a significantly stronger rich-club phenomenon than that obtained by the latter two models using the new-node-only growth. It is evident that the rich-club phenomenon is primarily determined by the growth mechanisms, not the preference schemes.

The BA model and the BA+PFP model use the newnode-only growth mechanism, in which all newly added 
TABLE II. Properties of the four models and the internet AS graph.

\begin{tabular}{lccccc}
\hline \hline & BA & IG & BA+PFP & PFP & AS graph \\
\hline Growth mechanism & New-node-only & $\mathrm{IG}_{p=0.4}$ & New-node-only & $\mathrm{IG}_{p=0.4}$ & \\
Preference scheme & Linear & Linear & $\mathrm{PFP}_{\delta=0.021}$ & $\mathrm{PFP}_{\delta=0.021}$ & \\
Number of nodes $N$ & 9204 & 9204 & 9204 & 9204 & 9204 \\
Number of links $L$ & 27612 & 27612 & 27612 & 27612 & 28959 \\
Rich-club phenomenon & weak & strong & weak & strong & strong \\
Rich-club exponent, $\theta$ & -0.97 & -1.49 & -0.97 & -1.49 & -1.481 \\
Rich-club connectivity $\varphi(0.01)$ & $6.0 \%$ & $40.3 \%$ & $5.7 \%$ & $44.8 \%$ & $44.3 \%$ \\
Top clique size $n_{\text {clique }}$ & 2 & 15 & 4 & 16 & 16 \\
Degree distribution $P(1)$ & $0 \%$ & $25.8 \%$ & $0 \%$ & $26.2 \%$ & $26.5 \%$ \\
Degree distribution $P(2)$ & $0 \%$ & $33.7 \%$ & $0 \%$ & $33.7 \%$ & $30.2 \%$ \\
Degree distribution $P(3)$ & $39.8 \%$ & $10.5 \%$ & $43.7 \%$ & $10.5 \%$ & $14.8 \%$ \\
Degree distribution exponent $\gamma$ & -2.902 & -2.206 & -2.890 & -2.255 & -2.254 \\
Maximum degree $k_{\text {max }}$ & 240 & 677 & 898 & 1950 & 2070 \\
Disassortative mixing & neutral & weak & weak & strong & strong \\
Assortativity coefficient $\alpha$ & -0.036 & -0.124 & -0.091 & -0.234 & -0.236 \\
Characteristic path length $\ell^{*}$ & 4.25 & 3.55 & 3.75 & 3.07 & 3.12 \\
\hline \hline
\end{tabular}

links are external links between new nodes and old nodes. The old nodes are preferentially chosen from rich nodes but the new nodes have only three initial links. Thus the only chance for the rich-club connectivity to increase is when new nodes become rich nodes, however, this usually happens in the early stage of network growth, and later on new nodes become more and more difficult to compete for new links against the already rich nodes. As a result, although rich nodes keep acquiring new links, the interconnections among rich nodes hardly increases.

In contrast the PFP model and the IG model use the interactive growth mechanism, which adds not only external links, but also internal links between old nodes. Since the old nodes are preferentially chosen from already rich nodes, the newly added internal links directly increase the rich-club connectivity. The ratio of internal links to external links can be estimated as a function of the parameter $p$,

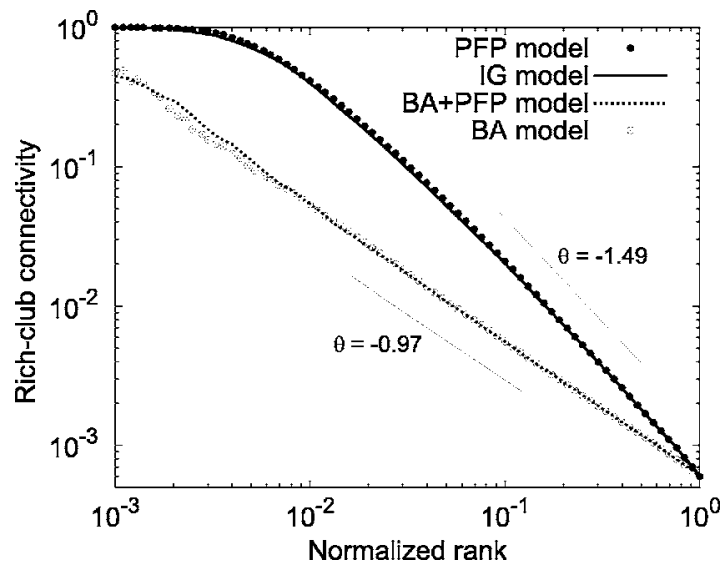

FIG. 4. Rich-club connectivity vs normalized rank, $\varphi(r / N)$.

$$
\frac{L_{i n t}}{L_{\text {ext }}}=\frac{2 p+(1-p)}{p+2(1-p)}=\frac{1+p}{2-p} .
$$

Simulation results show that when $p=0.4$, i.e., $L_{\text {int }} / L_{\text {ext }}$ $=7 / 8$, the interactive growth produces a rich-club phenomenon that precisely matches that of the internet AS graph.

The reason that using different preference schemes has little impact on the obtained rich-club phenomenon is that, although preference schemes influence the degree growth rate (i.e., how many links rich nodes have), they have little effect on the interconnectivity among the rich nodes.

\section{B. Degree distribution properties}

\section{Distribution of low degrees}

Table II and Fig. 5 show that more than half of the nodes

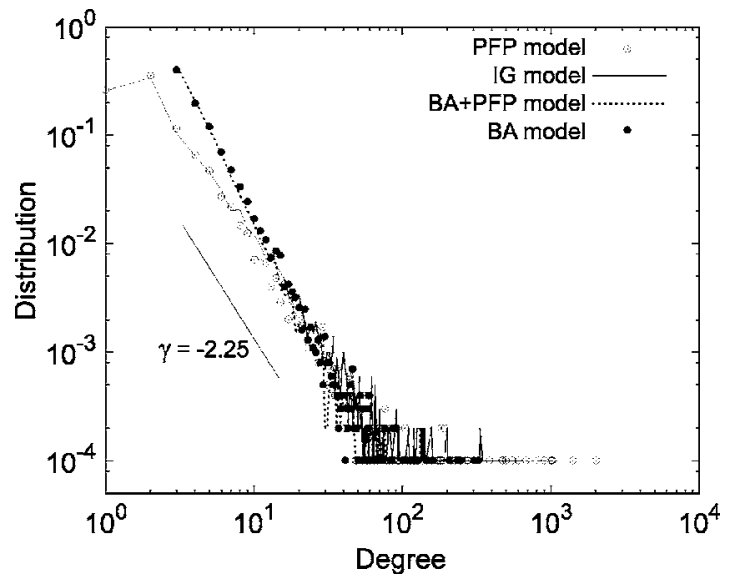

FIG. 5. Degree distribution $P(k)$. 


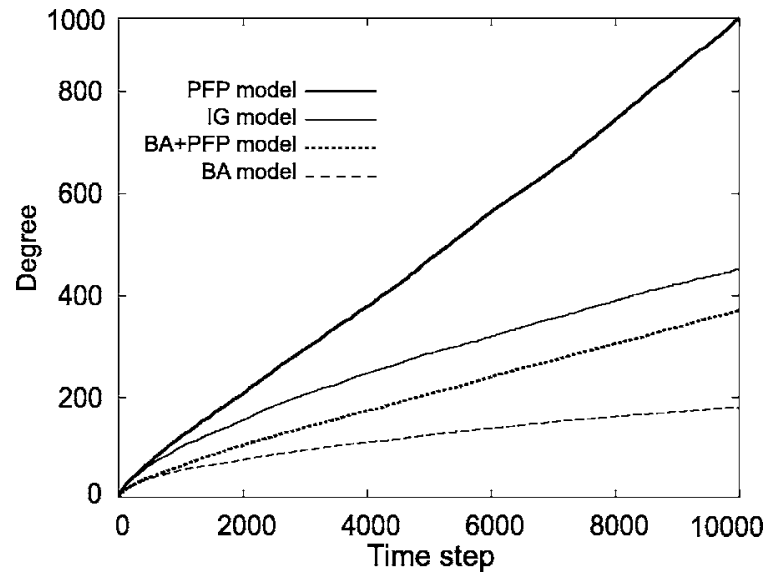

FIG. 6. Average degree growth of the initial nodes.

on the internet AS graph are one-degree or two-degree nodes, and the distribution of low degrees does not follow a power law because $P(k=1)=26.5 \%<P(k=2)=30.2 \%$. The BA model and the BA+PFP model do not contain one-degree and two-degree nodes because they use the new-node-only growth, in which each new node is attached to three old nodes, i.e., $k \geqslant 3$. The IG model and the PFP model use the interactive growth mechanism, which assigns a new node's initial degree as one or two according to the probability parameter $p$. Simulation results show that when $p=0.4$ the interactive growth closely resembles the AS graph's distribution of low degrees as well.

\section{Maximum degree and degree growth rate}

As shown in Fig. 5, the AS graph's degree distribution does not follow a strict power law because it has a heavy tail. The maximum degree $k_{\max }$ is the largest degree in a network. The maximum degree is an indicator of how far the degree distribution deviates from the prediction of a strict power law. Table II shows that the internet AS graph features a very large maximum degree, which is one order of magnitude larger than that generated by the BA model.

Figure 6 illustrates the growth of average degree of nodes contained in the initial small random graphs from which the networks grow. These nodes are the earliest ones present in the networks and usually they represent a sample of the rich nodes in the generated networks. As expected, the nodes in the BA+PFP model enjoy a higher degree growth rate than the BA model because the positive-feedback preference gives stronger preference to high-degree nodes than the BA model's linear preference.

Figure 6 also shows that the degree growth rate of the IG model is higher than the BA model and the BA + PFP model. This means the interactive growth is also able to accelerate the link acquiring process. As shown in Fig. 1, the new-nodeonly growth and the independent growth only allow a chosen old node to acquire one new link per time step, whereas the interactive growth is fundamentally different. It enables a chosen old node, i.e., a host node, to acquire up to three new links at each time step: with probability $p$, a host node acquires one external link and two internal links; and with probability $1-p$, a host node acquires one external link and

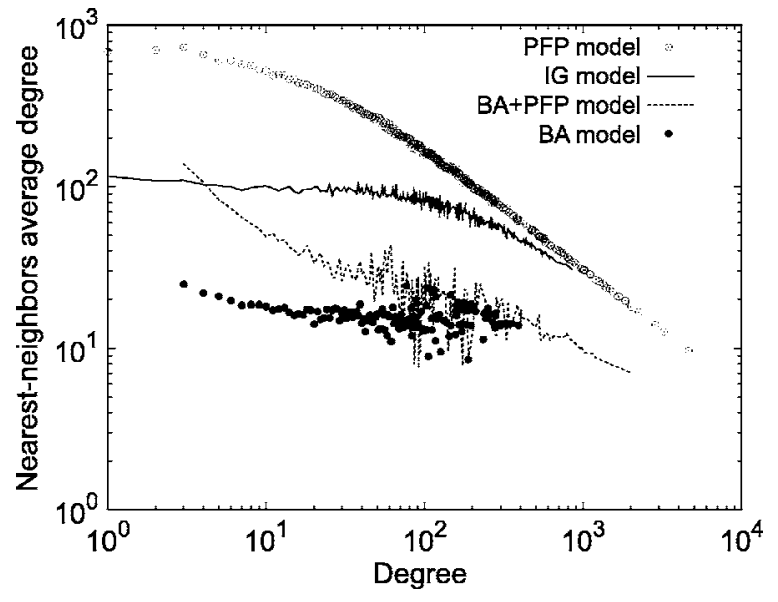

FIG. 7. Nearest-neighbors average degree vs node degree.

one internal link. We call this the degree-leap effect of the interactive growth. The degree-leap effect increases the degree of host nodes by 2 or 3 , which would take many time steps to achieve when using other growth mechanisms. The degree-leap effect also significantly enhances the node's ability to compete for even more new links in the forthcoming time steps, therefore the node's degree growth rate is accelerated.

The PFP model uses both the interactive growth and the positive-feedback preference, which reinforce each other, to achieve a degree growth rate that is notably more rapid than any other model. As a result, the PFP model obtains a very large maximum degree, as large as that of the internet AS graph.

\section{Power-law exponent of degree distribution}

As shown in Fig. 5, all four models produce power-law degree distributions, but have different power-law exponents, which result from fitting $P(k)$ in the area of $2 \leqslant k \leqslant 100$. Table II shows that the power-law exponents produced by the IG model and the PFP model, both using the interactive growth, are close to that of the internet AS graph, $\gamma=$ -2.254 , whereas the exponent generated by the BA model and the BA+PFP model is close to $\gamma=-3.0$ [1].

It is clear that the interactive growth has a major impact on the obtained degree distribution exponent. This is because the interactive growth has the degree-leap effect, which allows more nodes to be "fast tracked" into rich nodes and also makes the already rich nodes get richer more rapidly. The consequences of this dynamics is that the obtained powerlaw degree distribution exhibits a flatter slope (with a larger value of $\gamma$ ) and a heavier tail (with a larger maximum degree).

Studying the degree distribution exponents shown in Table II, we can see that the exponent of the IG model, $\gamma=$ -2.206 , is actually overly increased by the interactive growth and slightly larger than that of the internet AS graph. The PFP model accurately matches the AS graph's exponent because its positive-feedback preference has a minor effect of reducing the value of $\gamma$. This is because, comparing with the linear preference, the PFP gives a strong favor to the richest 


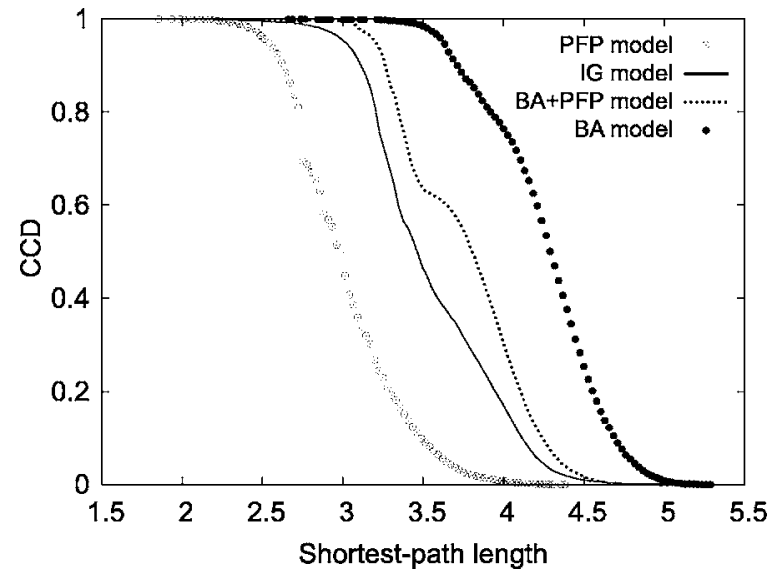

FIG. 8. Complimentary cumulative distribution (CCD) of shortest-path length $P(\geqslant \ell)$.

nodes (at the tail of the degree distribution) at the cost of all other nodes. As a result, the degree distribution is slightly steeper (with a smaller value of $\gamma$ ), the power law cuts off at a smaller degree, and the tail gets even longer.

\section{Disassortative mixing}

Networks exhibit different degree-degree mixing patterns [7-9]. For example, social networks are usually classified as assortative networks, because nodes in social networks statistically tend to attach to alike nodes, i.e., high-degree nodes to high-degree nodes and low-degree nodes to low-degree nodes. In contrast, technological and biological networks, e.g., the internet, exhibit the disassortative mixing, where high-degree nodes tend to connect with low-degree nodes, and vice versa. The BA model, however, is a neutral network that exhibits no mixing tendency.

The rich-club phenomenon observed on the internet does not conflict with the fact that the internet is a disassortative network, because the rich-club phenomenon does not imply that the majority links of the rich nodes are directed to other rich-club members. Indeed, rich nodes have very large numbers of links and only a few of them are enough to provide

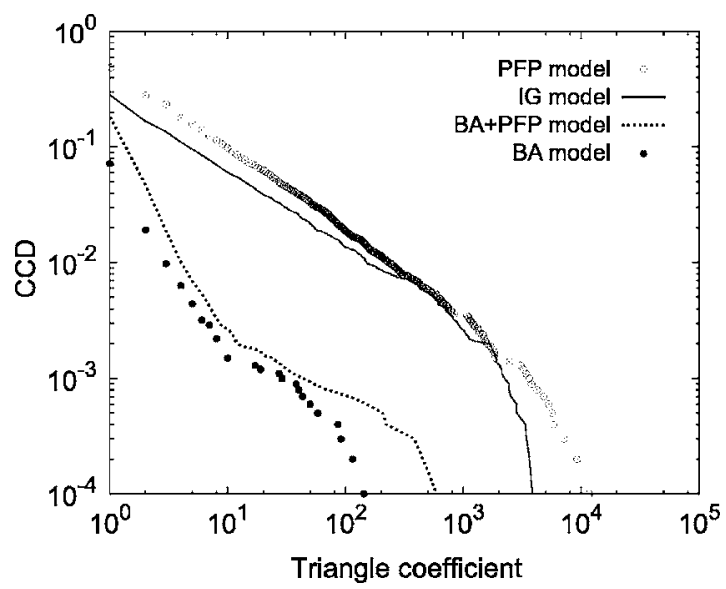

FIG. 9. Complement cumulative distribution of node triangle coefficient $P\left(\geq k_{t}\right)$.

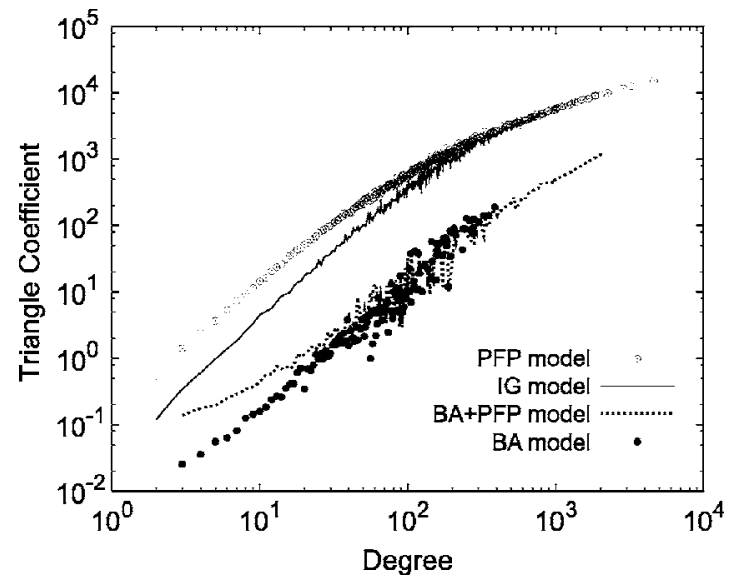

FIG. 10. Average triangle coefficient of $k$-degree nodes $k_{t}(k)$.

the interconnectivity to other club members, whose number is anyway small [2].

A network's mixing pattern is decided by the conditional probability $p_{c}\left(k^{\prime} \mid k\right)$ that a link connects a $k$-degree node to a $k^{\prime}$-degree node. For computational simplicity, a network's mixing pattern is often identified by the correlation between node degree and nearest-neighbors average degree [16]. As shown in Fig. 7, the PFP model is a disassortative network because it exhibits a negative degree-degree correlation, and the BA model is a neutral network because the nearestneighbors average degree is almost invariant to the node degree.

Another way to determine a network's mixing pattern is a metric called the assortativity coefficient [7], $\alpha \in[-1,1]$, which is defined as

$$
\alpha=\frac{L^{-1} \sum_{i} j_{i} k_{i}-\left[L^{-1} \sum_{i} \frac{1}{2}\left(j_{i}+k_{i}\right)\right]^{2}}{L^{-1} \sum_{i} \frac{1}{2}\left(j_{i}^{2}+k_{i}^{2}\right)-\left[L^{-1} \sum_{i} \frac{1}{2}\left(j_{i}+k_{i}\right)\right]^{2}},
$$

where $L$ is the number of links, and $j_{i}, k_{i}$ are the degrees of the nodes at the ends of the $i$ th link, with $i=1,2, \ldots, L$. When $\alpha>0$, a network is an assortative network, and when $\alpha<0$, it is a disassortative network. As shown in Table II, the internet AS graph, characterized by a negative assortativity coefficient of $\alpha=-0.236$, is closely resembled by the PFP model. The assortativity coefficient of the BA model is close to zero. In between are that of the IG model and the BA + PFP model.

This result shows that both the positive-feedback preference and the interactive growth contribute to a network's disassortative mixing behavior. As we have discussed in Sec. II $\mathrm{C}$, the positive-feedback preference can effectively amplify the degree difference between two nodes. Thus any degree difference between a link's two end nodes will be magnified by the PFP because the end node with a larger degree will grow faster and faster than the other end node. As a result the PFP increases a network's disassortative mixing. The interactive growth also increases the disassortative mixing but in a different way. When external links are attached 
TABLE III. The PFP model's sensitivity to parameter $p$ when $\delta=0$.

\begin{tabular}{lcccccc}
\hline \hline Interactive growth parameter $p$ & 0.0 & 0.2 & 0.4 & 0.6 & 0.8 & AS graph \\
\hline$L_{\text {int }} / L_{\text {ext }}=(1+p) /(2-p)$ & $1 / 2$ & $2 / 3$ & $7 / 8$ & $8 / 7$ & $3 / 2$ & \\
Rich-club exponent $\theta$ & -1.36 & -1.43 & -1.49 & -1.56 & -1.61 & -1.481 \\
Rich-club connectivity $\varphi(0.01)$ & $26.1 \%$ & $33.3 \%$ & $40.3 \%$ & $48.2 \%$ & $53.8 \%$ & $44.3 \%$ \\
Top clique size $n_{\text {clique }}$ & 7 & 10 & 15 & 17 & 20 & 16 \\
Degree distribution $P(1)$ & $0 \%$ & $13.1 \%$ & $25.8 \%$ & $38.9 \%$ & $50.5 \%$ & $26.5 \%$ \\
Degree distribution $P(2)$ & $49.5 \%$ & $41.6 \%$ & $33.7 \%$ & $25.9 \%$ & $18.7 \%$ & $30.2 \%$ \\
Degree distribution $P(3)$ & $13.5 \%$ & $12.1 \%$ & $10.5 \%$ & $8.4 \%$ & $6.6 \%$ & $14.8 \%$ \\
Degree distribution exponent $\gamma$ & -2.416 & -2.229 & -2.206 & -2.151 & -2.055 & -2.254 \\
Maximum degree $k_{\text {max }}$ & 573 & 625 & 677 & 722 & 763 & 2070 \\
Assortativity coefficient, $\alpha$ & -0.075 & -0.095 & -0.124 & -0.150 & -0.183 & -0.236 \\
Characteristic path length $\ell^{*}$ & 3.65 & 3.60 & 3.55 & 3.52 & 3.48 & 3.12 \\
& & & & & & \\
\hline \hline
\end{tabular}

between new nodes and host nodes, the host nodes are preferentially chosen from already rich nodes, and they enjoy the extra degree growth given by the interactive growth's degree-leap effect. However, the new nodes are to remain as low-degree, poor nodes. Thus the interactive growth introduces external links with a larger degree gap between the end nodes than other growth mechanisms do.

As shown in the IG model and the BA+PFP model, when either the interactive growth or the positive-feedback preference works alone, their effect on strengthening the disassortative mixing is limited. When the two mechanisms work together, as shown in the PFP model, they reinforce each other and generate networks as disassortative as the internet AS graph.

Up to this point, we have identified the explicit evolution dynamics that are responsible for the above topology properties. We can see the origin of these topology properties are embedded in the PFP model's two evolution mechanisms.

\section{Shortest path length}

The internet is a small-world network [10] because it is possible to get to any node via only a few links among adjoining nodes. The shortest path length $\ell$ is the minimum hop distance between a pair of nodes. Performance of modern network routing algorithms depends strongly on the distribution of shortest path length [24]. The characteristic path length $\ell^{*}$ is the average shortest path length over all pairs of nodes.

The characteristic path length of the internet AS graph is only 3.12 hops. The internet is so small because it exhibits both a strong rich-club phenomenon and a strong disassortative mixing. These two structural properties together contribute to the routing efficiency of the network. The rich club consists of a small number of highly connected nodes. The club members are tightly interconnected between each other. If two club members do not have a direct connection, they are very likely to share a neighboring club member. Thus the average hop distance between the members is very small (between 1 and 2 hops). The rich club functions as a "super" traffic hub of a network by providing a large selection of shortcuts for routing. The disassortative mixing property ensures that the majority of network nodes, peripheral lowdegree nodes, are always near the rich club. Thus a typical shortest path between two peripheral nodes consists of three hops, the first hop is from the source node to a member of the rich club, the second hop is between two club members, and the final hop is to the destination node.

As shown in Fig. 8 and Table II, the PFP model accurately reproduces the rich-club phenomenon and the disassortative mixing of the internet AS graph; naturally it reproduces the internet's shortest path length as well. The BA model exhibits a weak rich-club phenomenon and it is a neutral network. As a result the BA model's characteristic path length is more than one hop longer than that of the internet AS graph. This one-hop difference is significant considering that the AS graph's characteristic path length is merely over three hops. The BA+PFP model exhibit a rich-club phenomenon as

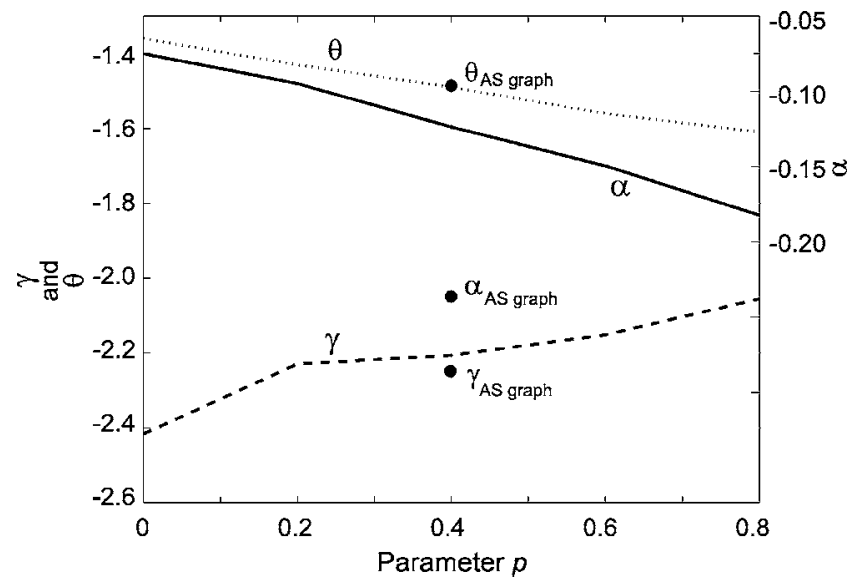

FIG. 11. Properties of the PFP model when parameter $p$ grows from 0 to 0.8 while $\delta=0$, including the degree distribution exponent $\gamma$, the rich-club connectivity exponent $\theta$, and the assortativity coefficient $\alpha$. 
TABLE IV. The PFP model's sensitivity to parameter $\delta$ when $p=0.4$.

\begin{tabular}{lccccccc}
\hline \hline Positive-feedback parameter $\delta$ & 0 & 0.007 & 0.014 & 0.021 & 0.028 & 0.035 & AS graph \\
\hline Rich-club exponent $\theta$ & -1.49 & -1.49 & -1.49 & -1.49 & -1.50 & -1.44 & -1.481 \\
Rich-club connectivity $\varphi(0.01)$ & $40.3 \%$ & $44.0 \%$ & $43.5 \%$ & $44.8 \%$ & $46.4 \%$ & $36.5 \%$ & $44.3 \%$ \\
Top clique size, $n_{\text {clique }}$ & 15 & 16 & 16 & 16 & 20 & 15 & 16 \\
Degree distribution $P(1)$ & $26.2 \%$ & $26.4 \%$ & $26.8 \%$ & $26.2 \%$ & $26.4 \%$ & $22.0 \%$ & $26.5 \%$ \\
Degree distribution $P(2)$ & $33.7 \%$ & $34.3 \%$ & $34.8 \%$ & $33.7 \%$ & $35.2 \%$ & $34.7 \%$ & $30.2 \%$ \\
Degree distribution $P(3)$ & $10.5 \%$ & $10.6 \%$ & $10.5 \%$ & $10.5 \%$ & $12.4 \%$ & $16.7 \%$ & $14.8 \%$ \\
Degree distribution exponent $\gamma$ & -2.206 & -2.219 & -2.228 & -2.255 & -2.321 & -2.540 & -2.254 \\
Maximum degree $k_{\max }$ & 677 & 875 & 1356 & 1950 & 2519 & 7045 & 2070 \\
Assortativity coefficient, $\alpha$ & -0.124 & -0.172 & -0.202 & -0.234 & -0.279 & -0.292 & -0.236 \\
Characteristic path length $\ell^{*}$ & 3.55 & 3.41 & 3.24 & 3.07 & 2.93 & 2.44 & 3.12 \\
\hline \hline
\end{tabular}

weak as the BA model, but it exhibits a (weak) disassortative mixing. Consequently its characteristic path length is a half hop shorter than that of the BA model. The IG model is more disassortative than the BA+PFP model, and it exhibits the rich-club phenomenon as strong, as the PFP model. Therefore the IG model is smaller than the BA+PFP model, but not as small as the PFP model.

\section{E. Short cycles-triangle coefficient}

Short cycles, i.e., triangles and quadrangles, encode the redundancy information in a network because the multiplicity of paths between any two nodes increases with the density of short cycles $[11,12]$. The triangle coefficient $k_{t}$ is defined as the number of triangles that a node shares, or the number of links connecting among a node's nearest neighbors. The clustering coefficient [10] can be given as $c=\frac{k_{t}}{k(k-1) / 2}$. Comparing with the clustering coefficient, the triangle coefficient is able to infer neighbor clustering information of nodes with different degrees, i.e., the correlation between triangle coefficient and degree.

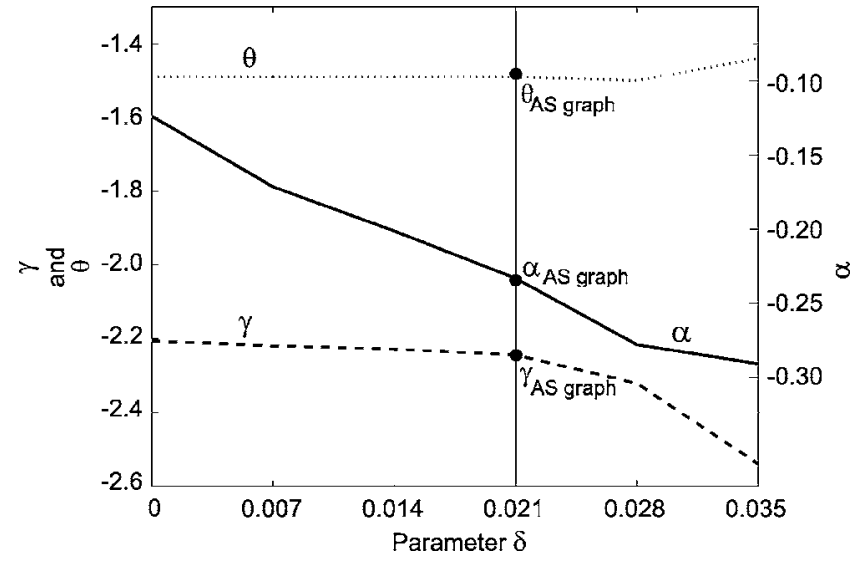

FIG. 12. Properties of the PFP model when parameter $\delta$ grows from 0 to 0.035 while $p=0.4$, including the degree distribution exponent $\gamma$, the rich-club connectivity exponent $\theta$, and the assortativity coefficient $\alpha$.
Figure 9 shows that the density of triangles of the PFP model and the IG model is significantly larger than that of the BA model and the BA+PFP model. Figure 10 shows that the average triangle coefficient of $k$-degree nodes in the PFP model and the IG model is one order of magnitude larger than that of nodes with the same degrees in the BA model and the BA+PFP model. It is evident that models showing a stronger rich-club phenomenon contain considerably more triangles. This is because the interconnections between richclub members play a major role on the formation of triangles, not only for the club members themselves, but also for the peripheral low-degree nodes which have more than one connections to the rich club.

The disassortative mixing also has a minor impact on the formation of triangles. When a model exhibits a stronger disassortative mixing, links of the low-degree nodes are more likely connected to the rich club, and thus form more triangles. For example, the PFP model and the IG model show the same rich-club phenomenon but the PFP model exhibits a stronger disassortative mixing. Thus, as shown in Fig. 10, the average triangle coefficient of nodes in the degree area of $2<k<100$ in the PFP model is notably larger than that of nodes with the same degrees in the IG model.

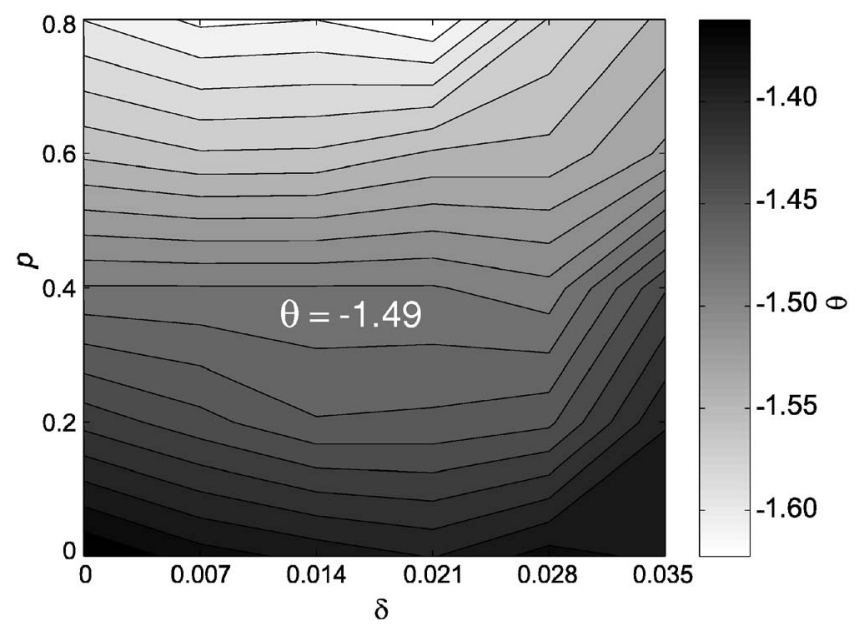

FIG. 13. Rich-club connectivity's power-law exponent $\theta$ vs parameters $p$ and $\delta$. 


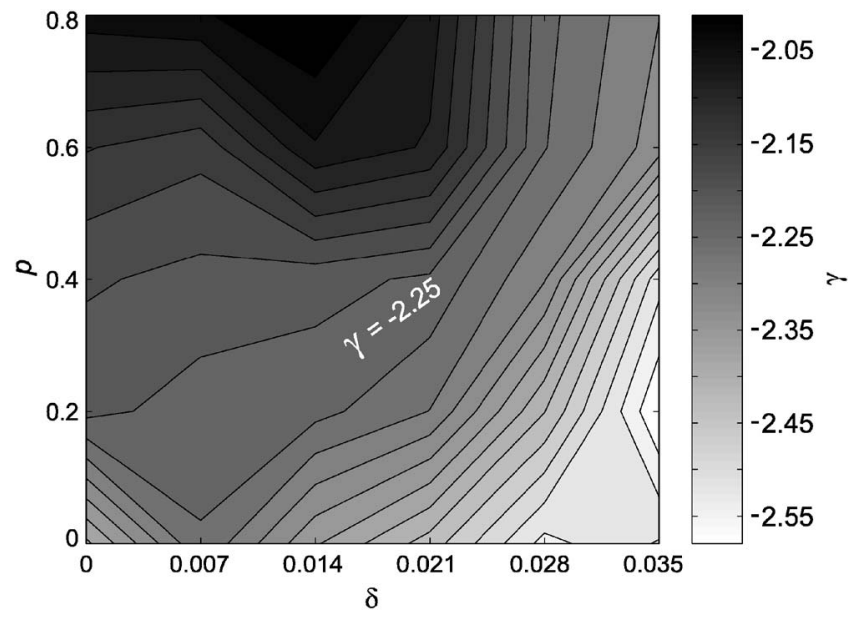

FIG. 14. Degree distribution's power-law exponent $\gamma$ vs parameters $p$ and $\delta$.

The same can be observed by contrasting the BA+PFP model against the $\mathrm{BA}$ model in the degree area of $3<k$ $<30$.

In summary, the PFP model captures the internet's shortest path length and triangle coefficient because these properties are correlated with other topology properties that have been produced by the model's evolution mechanisms.

\section{SENSITIVITY TO MODEL PARAMETERS}

The PFP model's interactive growth and positivefeedback preference are controlled by the parameters $p$ and $\delta$, respectively. In the previous section the PFP model uses $p=0.4$ and $\delta=0.021$ to generate internetlike networks. In this section we provide more detailed numerical results to support the above analysis. We examine how the parameters control the evolution mechanisms and therefore change the generated topology properties. We first study the model's sensitivity to individual parameters by making one parameter a variable and fixing the other as a constant. Then we investigate the model's reactions when both parameters are variables.

\section{A. Sensitivity to parameter $p$}

Table III and Fig. 11 show how topology properties of the PFP model change when the interactive growth parameter $p$ increases from 0 to 0.8 while the positive-feedback parameter $\delta=0$ (i.e., equivalent to the linear preference). It is clear that when $p$ increases, the rich-club phenomenon is getting stronger and stronger as the value of rich-club exponent $\theta$ decreases monotonically. This is because the ratio of new internal links to new external links added to the model increases with $p$ [see Eq. (4)]. When more internal links are added, the rich nodes become more tightly interconnected. Also the interactive growth has a direct impact on the distribution of low degrees. When $p$ increases, the generated network contains more one-degree nodes and less two-degree nodes [see $P(1)$ and $P(2)$ in Table III]. When $p=0.4$, the interactive growth well resembles the internet AS graph's

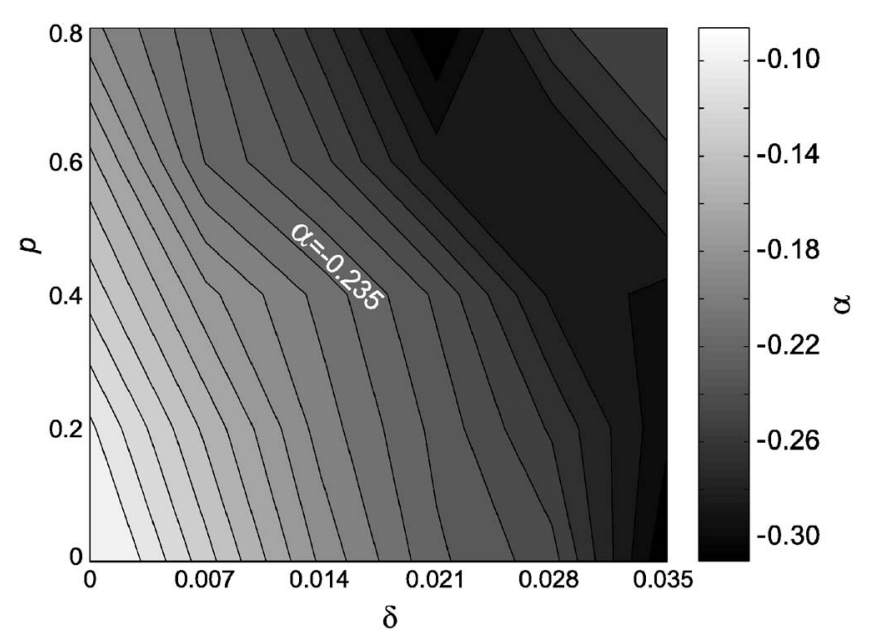

FIG. 15. Assortativity coefficient $\alpha$ vs parameters $p$ and $\delta$.

rich-club phenomenon and distribution of low degrees at the same time.

As analyzed in the above, the interactive growth has a degree-leap effect. When $p$ increases, more three-degree leaps [see Fig. 1(c)] and less two-degree leaps [see Fig. 1(d)] take place during the network growth. Thus the overall degree-leap effect become stronger. As a result the powerlaw degree distribution becomes flatter with an increased value of the degree distribution exponent $\gamma$. As shown in Fig. 11 , when $p$ increases to $0.4, \gamma$ exceeds that of the internet AS graph. We will see in the next section that this excessive increase of $\gamma$ will be counterbalanced by the positivefeedback preference's opposite effect on $\gamma$.

When $p$ grows from 0 to 0.8 , the strengthened degree-leap effect also enlarges the obtained maximum degree, and strengthens the model's disassortative mixing behavior indicated by a decreasing value of the assortativity coefficient. As expected, when the rich-club phenomenon and the disassortative mixing become stronger, the generated networks get smaller and smaller as indicated by a decreasing value of the characteristic path length. However, when $p=0.8$ and the model exhibits a stronger rich-club phenomenon than the internet AS graph, the PFP model's characteristic path length is still not as small as the internet. This is because the model's disassortative mixing is as strong as the internet. In order to resemble the internet, the interactive growth needs to be combined with the positive-feedback preference.

\section{B. Sensitivity to parameter $\delta$}

Table IV and Fig. 12 show how topology properties of the PFP model change when the positive-feedback parameter $\delta$ increases from 0 to 0.035 while the interactive growth parameter $p=0.4$. It is clear that the positive-feedback parameter $\delta$ has a fairly limited impact on the obtained rich-club exponent $\theta$, which almost remains the same. When $\delta$ increases, greater preference is given to high-degree nodes; consequently the maximum degree increases and the network becomes more disassortative mixed. As expected, the characteristic path length decreases steadily when the network's disassortative mixing is getting stronger. When the parameter 
$\delta$ increases, the degree distribution power-law exponent $\gamma$ slightly decreases. This is because when the positivefeedback preference gets stronger, the richest nodes attract so many new links that they suppress the degree growth of other nodes, including those with medium to high degrees. When $\delta=0.021$ (and $p=0.4$ ), the PFP model closely matches all the topology properties of the internet AS graph.

As shown in Fig. 12, by tuning the parameter $\delta$ from 0 to 0.028, the PFP model is capable of generating a wide range of disassortative mixing networks with the value of the assortativity coefficient $\alpha$ decreasing monotonically from -0.12 to -0.28 , which encompasses most technological and biological networks reported in Ref. [25]. Notably, the PFP model achieves this while keeping the rich-club exponent and the degree distribution exponent largely unchanged.

$\delta=0.028$ is the model's tipping point, beyond which the network structure changes dramatically, e.g., the degree distribution exponent $\gamma$ decreases rapidly. This is because the preferential selection becomes so biased that super-rich nodes start to emerge. These extremely rich nodes dominate the network growth and make the network grow towards a starlike structure.

\section{Sensitivity to both parameters}

Figures 13-15 are contour plots showing how three topology properties, i.e., the rich-club connectivity exponent $\theta$, the degree distribution exponent $\gamma$, and the assortativity coefficient $\alpha$, change when both the parameters are variables. Figure 13 clearly shows that the value of rich-club exponent $\theta$ is sensitive to the interactive growth parameter $p$ and is unsensitive to the positive-feedback preference parameter $\delta$. When parameter $p$ grows, the rich-club phenomenon becomes stronger. Figure 14 shows that the two mechanisms have opposite effects on the value of degree distribution exponent $\gamma$. In general, the exponent $\gamma$ increases when parameter $p$ increases and parameter $\delta$ decreases.

Figure 15 shows that the PFP model's disassortative mixing becomes stronger when either of the two parameters increases. The assortativity coefficient $\alpha$ is more sensitive to parameter $\delta$ than to parameter $p$. We notice that when $p$ $>0.5$, the network's disassortative mixing actually becomes weaker. This is because, when $p>0.5$, the network starts to acquire more internal links than external links, i.e., $L_{\text {int }} / L_{\text {ext }} \geq 1$ [see Eq. (4)]. The increased new internal links among the rich nodes weaken the disassortative mixing behavior of the network.

\section{CONCLUSION}

The internet has an extremely complex structure. The PFP model demonstrates a way to simultaneously reproduce many topology properties of the internet. The model achieves this by using two generic algorithms which are designed to replicate the evolution dynamics observed on the internet historic data. In this paper we have used the numerical method to investigate the success of the model.

Based on detailed simulation results, we show that the rich-club phenomenon is primarily controlled by the interactive growth mechanism alone. We point out that this is because the rich-club connectivity increases with the number of new internal links which are added between rich nodes. The interactive growth also determines the probability of new nodes' initial degrees and thus controls the distribution of low degrees obtained in the generated network.

The PFP model's maximum degree is as large as the internet because both the interactive growth and the positivefeedback preference accelerate the degree growth rate. The interactive growth does so by the degree-leap effect, whereas the positive-feedback preference achieves so by giving stronger preference to high-degree nodes. The two mechanisms have opposite effects on the value of power-law exponent of degree distribution. The interactive growth increases the value whereas the positive-feedback preference slightly decreases the value.

We have explained that the origin of the disassortative mixing has been, unintentionally, embedded in the PFP model's two evolution mechanisms, which not only enlarge the gap between the high-degree nodes and the low-degree nodes, but more critically, they increase the degree difference between end nodes of a link.

Our analysis suggests that the reason that the PFP model also captures other properties of the internet, such as shortest path length and triangle coefficient, is that these properties are correlated with other properties, in other words, they are constrained by others. For example, the internet is small because the rich-club functions as a super traffic hub, while the disassortative mixing behavior ensures peripheral low-degree nodes always close to the hub.

By investigating the PFP model's sensitivity to the mechanism parameters, we obtain more evident details on how the two evolution mechanisms profoundly control the model's growth dynamics and therefore effectively change the generated topology properties. For example, we show that by tuning the positive-feedback parameter $\delta$, the PFP model is able to generate a wide range of disassortative mixing networks while keeping the rich-club exponent and the degree distribution exponent almost unchanged.

This work represents a better understanding of the internet topology evolution dynamics. It facilitates the ongoing effort on a mathematical analysis of the PFP model. This work also complements the research on the evolution dynamics and the structural constraints of complex networks in general. 
[1] R. Albert and A. L. Barabási, Rev. Mod. Phys. 74, 47 (2002).

[2] R. Pastor-Satorras and A. Vespignani, Evolution and Structure of the Internet-A Statistical Physics Approach (Cambridge University Press, Cambridge, England, 2004).

[3] S. Floyd and E. Kohler, Comput. Commun. Rev. 33, 29 (2003).

[4] S. Zhou and R. J. Mondragón, Phys. Rev. E 70, 066108 (2004).

[5] S. Zhou and R. J. Mondragón, IEEE Commun. Lett. 8, 180 (2004).

[6] M. Faloutsos, P. Faloutsos, and C. Faloutsos, Comput. Commun. Rev. 29, 251 (1999).

[7] M. E. J. Newman, Phys. Rev. Lett. 89, 208701 (2002).

[8] A. Vazquez, M. Boguna, Y. Moreno, R. Pastor-Satorras, and A. Vespignani, Phys. Rev. E 67, 046111 (2003).

[9] S. Maslov, K. Sneppen, and A. Zaliznyak, Physica A 333, 529 (2004).

[10] D. J. Watts and S. H. Strogatz, Nature (London) 393, 440 (1998)

[11] G. Bianconi and A. Capocci, Phys. Rev. Lett. 90, 078701 (2003).

[12] G. Caldarelli, R. Pastor-Satorras, and A. Vespignani, Eur. Phys. J. B 28, 183 (2004).

[13] K. I. Goh, B. Kahng, and D. Kim, Phys. Rev. Lett. 87, 278701 (2001).

[14] US National Science Foundation (NSF) Project No. 0434996 (2004), http://www.caida.org/funding/nets-nr/

[15] A. L. Barabási and R. Albert, Science 286, 509 (1999).

[16] A. Vázquez, R. Pastor-Satorras, and A. Vespignani, Phys. Rev.
E 65, 066130 (2002).

[17] Q. Chen, H. Chang, R. Govindan, S. Jamin, S. J. Shenker, and W. Willinger, in Proceedings of IEEE INFOCOM 2002 (IEEE Computer Society, Washington D.C., 2002), pp. 608-617.

[18] S. T. Park, A. Khrabrov, D. M. Pennock, S. Lawrence, C. L. Giles, and L. H. Ungar, in Proceedings of IEEE INFOCOM 2003 (IEEE Computer Society, Washington D.C., 2003), pp. 2144-2154.

[19] T. Bu and D. Towsley, in Proceedings of IEEE INFOCOM 2002 (IEEE Computer Society, Washington D.C., 2002), pp. 638-647.

[20] S. N. Dorogovtsev and J. F. F. Mendes, Europhys. Lett. 52, 33 (2000).

[21] P. L. Krapivsky, S. Redner, and F. Leyvraz, Phys. Rev. Lett. 85, 4629 (2000).

[22] ITDK0304, CAIDA's Internet AS-level topology data kit collected in April 2003. http://www.caida.org/tools/measurement/ skitter/idkdata.xml

[23] P. Mahadevan, D. Krioukov, M. Fomenkov, B. Huffaker, X. Dimitropoulos, and K. Claffy, Comput. Commun. Rev. 36, 17 (2006).

[24] C. Labovitz, A. Ahuja, R. Wattenhofer, and S. Venkatachary, in Proceedings of IEEE INFOCOMM 2001 (IEEE Computer Society, Washington D.C., 2001), pp. 537-546.

[25] M. E. J. Newman, Phys. Rev. E 67, 026126 (2003).

[26] Different from Ref. [4], in this paper we use the natural logarithm in Eq. (3) of the positive-feedback preference. Accordingly the value of parameter $\delta$ is revised as $\delta_{\text {new }}=\delta_{\text {old }} / \ln 10$. 